\title{
Reshaping the binding channel of a novel GH113 family $\beta$-Mannanase from Paenibacillus cineris (PcMan113) for enhanced activity
}

\section{Dengyue Sun}

Qilu University of Technology

Chao Li

Tianjin University of Science and Technology; Tianjin 300457, P. R. China

Pengpeng Cui

Qilu University of Technology, Jinan 250353, Shandong Province, PR China

Jie Zhang

Qilu University of Technology, Jinan 250353, Shandong Province, PR China

\section{Yaolin Zhou}

Qilu University of Technology, Jinan 250353, Shandong Province, PR China

\section{Mian Wu}

Tianjin University of Science and Technology; Tianjin 300457, P. R. China

\section{Xia Li}

Qilu University of Technology, Jinan 250353, Shandong Province, PR China

\section{Teng-Fei Wang}

Qilu University of Technology; Shandong Academy of Sciences; Jinan 250100, P. R. China

\section{Zhixiong Zeng}

Qilu University of Technology, Jinan 250353, Shandong Province, PR China

Hui-Min Qin ( $\square$ huiminqin@tust.edu.cn )

Tianjin University of Science and Technology; Tianjin 300457, P. R. China

\section{Research}

Keywords: Endo- $\beta-M a n n a n a s e, ~ G H 113$ family, Structural analysis, Binding channel, Molecular dynamics

Posted Date: October 18th, 2021

DOI: https://doi.org/10.21203/rs.3.rs-970728/v1

License: (c) (1) This work is licensed under a Creative Commons Attribution 4.0 International License. Read Full License 
Version of Record: A version of this preprint was published at Bioresources and Bioprocessing on March 5th, 2022. See the published version at https://doi.org/10.1186/s40643-022-00505-7. 


\section{Abstract}

Endo- $\beta$-mannanases are an important enzyme for degrading lignocellulosic biomass to generate mannan, which has significant health effects as a prebiotic that promotes the development of gut microbiota. Here, a novel endo- $\beta$-mannanase belonging to glycoside hydrolase (GH) family 113 from Paenibacillus cineris (PcMan113) was cloned, expressed and characterized, as one of only a few reported $\mathrm{GH} 113$ family $\beta$-mannanases. Compared to other functionally and structurally characterized $\mathrm{GH} 113$ mannanases, recombinant PcMan113 showed a broader substrate spectrum and a better performance. Based on a structural homology model, the highly active mutant PcMT3 (F110E/N246Y) was obtained, with 4.60- and 5.53-fold increases of enzyme activity (towards KG) and catalytic efficiency $\left(k_{\text {cat }} / K_{\mathrm{m}}\right.$, against M5) compared with the WT enzyme, respectively. Furthermore, molecular dynamics (MD) simulations were conducted to precisely explore the differences of catalytic activity between WT and PcMT3, which revealed that PcMT3 has a less flexible conformation, as well as an enlarged substrate binding channel with decreased steric hindrance and increased binding energy in substrate recognition. In conclusion, we obtained a highly active variant of PcMan113 with potential for commercial application in the manufacture of mannooligosaccharides.

\section{Introduction}

Hemicellulose is the second most abundant polysaccharide in nature, and mannan is a major hemicellulose component normally found in the cell walls and seeds of plants (Saha, 2003). The degradation of mannan polysaccharides relies on microbial mannanases, which are primarily endo-type enzymes, and the resulting manno-oligosaccharide (MOS) products are further degraded into monomers, which play important roles in microbial metabolism and plant growth (Srivastava et al. 2017; Srivastava et al. 2005; Moreira et al. 2008). Normally, MOS prepared by enzymatic methods are mainly sourced from locust bean gum (LBG), konjac gum (KG), and guar gum (GG) (Malgas et al. 2015).

Mannan is receiving increasing attention due to its health effects mediated by gut microbiota and probiotics (Mano et al. 2018; Pérez-Burillo et al. 2019). MOS act as prebiotics that can also be used in food directly, and have attracted attention from the healthcare industry due to their functional activities, such as promoting growth of probiotics, inhibiting the growth of pathogenic bacteria and contributing to the health of the intestinal environment (Pérez-Burillo et al. 2019; Zheng et al. 2018; Srivastava et al. 2014; Yamabhai et al. 2016; La Rosa et al. 2019). The practice of using MOS as feed additive can enhance the growth performance of pigs, as well as improve posterior gut epithelial defense, egg production and antibody production efficiency of laying hens (Giannenas et al. 2016; Ghasemian et al. 2016). In addition, MOS have been reported to enhance the immunity and resistance of Pacific white shrimp (Torrecillas et al. 2014). Therefore, the demand for MOS is increasing due to their great potential for applications in the feed, food, and pharmaceutical industries (Srivastava et al. 2017; Cao et al. 2018; Rai et al. 2012). 
MOS are generally produced via the enzymatic hydrolysis of LBG, KG, and GG due to the higher extraction efficiency, environmental benefits, lower cost, and easy operation (Yang et al. 2019). Based on present reports on the preparation of MOS, several enzymes, such as mannanases, mannosidases, galactosidases and glucosidases, must work in synergy to achieve the desired degree of polymerization

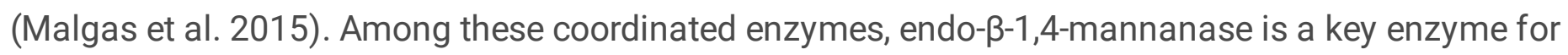
cutting off the random $\beta-1,4$-mannosidic linkages in mannan polysaccharides for MOS preparation (Srivastava et al. 2017).

Based on sequence similarities and structural properties, mannanases can be classified into glycoside hydrolase (GH) families 5, 26 and 113 (Zhang et al. 2008). These enzymes have distinct modes of substrate recognition, but the molecular mechanisms governing their substrate preference and mode of action are still not fully understood. The majority of currently used mannanase-producing microorganisms such as Bacillus sp. (Zhang et al. 2008; David et al. 2018; Eom et al. 2016), Klebsiella sp. (Pongsapipatana et al. 2016) and Aspergillus sp. (Shimizu et al. 2015) cannot meet the increasing demand for food-grade MOS products. Although a number of studies focused on the identification of new $\beta$-mannanases to prepare MOS from one or two kinds of mannans, there are only a few reports on $\beta$ mannanases belonging to the GH113 family that can effectively hydrolyze $\mathrm{LBG}, \mathrm{KG}$, and $\mathrm{GG}$ for the preparation of MOS (Cao et al. 2018; Yang et al. 2019; Yang et al. 2015; Li et al. 2017). Furthermore, finding mannanases with high activity and stability during the catalytic process is essential for industrial application in MOS production. Therefore, mannanases which have specific characteristics, such as higher activity, acid resistance and thermal stability, are currently required (Yang et al. 2019).

In this work, we focused on a novel mannanase with higher catalytic activity and thermostability, which could potentially be used for industrial MOS production. We fully characterized its enzymological characteristics relevant for industrial use, and identified its key catalytic active site residues via homologous modeling and structural analysis of PcMan113. Rational design of enzyme for structurebased engineering was applied for obtaining the highly active mutant PcMT3 with desired properties. Subsequently, molecular docking and molecular dynamics (MD) simulations were then conducted to identify and explain the differences between highly active mutants and the wild-type enzyme. Our work provides the significantly improved variant PCMT3 for potential commercial use in the food and feed industries.

\section{Materials And Methods}

\section{Materials}

The restriction enzymes, PrimeSTAR MAX DNA polymerase, and T4 DNA ligase were obtained from TaKaRa Biotechnology (Dalian, China). LBG, KG, GG and standard chemicals were purchased from Sigma-Aldrich (St. Louis, MO, USA) or Yuanye (Shanghai, China) and were of analytical grade unless stated otherwise. MOS standards (M2-M6) were purchased from Megazyme (Wicklow, Ireland). Synthetic oligonucleotides and primers were obtained from Genewiz (Suzhou, China). 


\section{Vector construction and expression of endo- $\beta-1,4-$ mannanase}

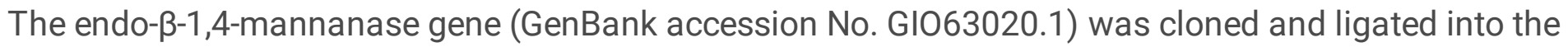
pQE-80L vector (Qiagen, Hilden, Germany) between the BamHI and Hindlll restriction sites, so that it contained a His6 tag $(\mathrm{HHHHHH})$ at the $N$-terminus. The recombinant plasmid harboring the mannanase sequence was introduced into $E$. coli BL21(DE3), which were grown in lysogeny broth (LB) at $37^{\circ} \mathrm{C}$. When the $\mathrm{OD}_{600}$ reached 0.8 , isopropyl $\beta$-D-1-thiogalactopyranoside (IPTG) was added to a final concentration of $0.5 \mathrm{mM}$ to induce protein overexpression, which was continued at $16^{\circ} \mathrm{C}$ overnight. After that, the cells were harvested by centrifugation at $4,000 \mathrm{~g}$ and $4^{\circ} \mathrm{C}$ for $15 \mathrm{~min}$, and resuspended in lysis buffer $(20 \mathrm{mM}$ Tris- $\mathrm{HCl}, \mathrm{pH}$ 8.0, $20 \mathrm{mM}$ imidazole, $0.5 \mathrm{M} \mathrm{NaCl}$ and $1 \mathrm{mM}$ dithiothreitol). The cells were disrupted by sonication with 2 s pulse, 2 s output and $50 \%$ duty cycle for 30 min on ice.

\section{Protein purification and determination of molecular mass}

The His-tag target protein was trapped onto $2 \mathrm{~mL}$ of Ni-NTA Superflow resin (Qiagen, Hilden, Germany) pre-equilibrated with $20 \mathrm{~mL}$ of lysis buffer. After washing the resin with buffer $(20 \mathrm{mM}$ Tris-HCl, pH 8.0, 40 $\mathrm{mM}$ imidazole, $0.5 \mathrm{M} \mathrm{NaCl}$ and $1 \mathrm{mM} \mathrm{DTT}$ ), the target protein was eluted with $10 \mathrm{~mL}$ of elution buffer (20 $\mathrm{mM}$ Tris- $\mathrm{HCl}, \mathrm{pH} 8.0,500 \mathrm{mM}$ imidazole, $0.5 \mathrm{M} \mathrm{NaCl}$ and $1 \mathrm{mM} \mathrm{DTT}$ ) at $4^{\circ} \mathrm{C}$. The eluted fractions containing the target protein were pooled and concentrated by ultrafiltration (10 kDa MWCO, Millipore, Billerica, MA, USA) at $4^{\circ} \mathrm{C}$, and further purified by gel filtration using a Superdex $200 \mathrm{HR} 10 / 300$ column (GE Healthcare, Uppsala, Sweden). Fractions containing recombinant mannanase were concentrated by ultrafiltration at $4^{\circ} \mathrm{C}$. The purity and molecular mass of the purified mannanase was determined by sodium dodecyl sulfate polyacrylamide gel electrophoresis (SDS-PAGE) (Zhao et al. 2012). The purified protein was concentrated by ultrafiltration, and used for activity assays.

\section{Mannanase activity assay}

Mannanase activity was measured using the previously described method of 3,5-dinitrosalicylic acid (DNS) colorimetric reported by $\mathrm{Li}$ and co-workers and with slight modification, and $0.5 \%\left(\mathrm{wv}^{-1}\right) \mathrm{LBG}$ was chosen as the substrate (Akino et al. 1988). One unit of enzyme activity was defined as the amount of enzyme that converts $1 \mu \mathrm{mol}$ substrate per min under the described assay conditions. The absorbance of colored solution was monitored at $540 \mathrm{~nm}$ by an ultraviolet-visible (UV-vis) spectrophotometer (Jinghua, Shanghai, China). Kinetic studies of wild-type (WT) mannanase and mutants were conducted in the same reaction system. The fitting curves of WT and mutants with LBG, GG and KG as substrates were recorded, and the $K_{\mathrm{m}}$ and $k_{\text {cat }}$ parameters were calculated using nonlinear fitting to the Michaelis-Menten equation in GraphPad Prism 7.0 (GraphPad software, La Jolla, CA). The protein concentration was determined according to the Lowry method (Lowry et al, 1951), with bovine serum albumin (BSA) as the standard.

\section{Characterization of PcMan113}


The optimal pH of the purified PcMan113 was determined by measuring the enzyme's activity in $25 \mathrm{mM}$ $\mathrm{Na}_{2} \mathrm{HPO}_{4}$-citrate buffer ( $\mathrm{pH}$ 3.0-6.0), $50 \mathrm{mM}$ sodium acetate-acetic acid buffer ( $\mathrm{pH}$ 4.5-5.5), $50 \mathrm{mM}$ phosphate-citric acid buffer (pH 6.0-8.0), $25 \mathrm{mM}$ Tris-HCl buffer (pH 7.0-9.0) and $25 \mathrm{mM}$ glycine-NaOH buffer (pH9.5-10.5) at $25^{\circ} \mathrm{C}$ for $5 \mathrm{~min}$. The optimal temperature was determined by incubating PcMan 113 at $15-65^{\circ} \mathrm{C}$. The thermostability was determined by measuring the residual activity after incubation of the enzyme at 20 to $85^{\circ} \mathrm{C}$ for $30 \mathrm{~min}$. The effect of $\mathrm{pH}$ on protein stability was tested by incubating the purified PcMan 113 protein at different $\mathrm{pH}$ values $\left(\mathrm{pH}\right.$ 5.0-9.0) for $1 \mathrm{~h}$ at $4^{\circ} \mathrm{C}$. The activity was then measured under standard reaction conditions. LBG, cassia gum, guar gum, and konjac powder were used to assess the substrate specificity of PcMan113. All activity assays were carried out in triplicate.

\section{Analysis of hydrolysis products}

For the production of manno-oligosaccharides LBG, KG, and GG were used as the substrates, respectively, and were incubated with $5 \mu \mathrm{M}$ of purified protein at $60^{\circ} \mathrm{C}$ and $\mathrm{pH} 5.0$ for $10 \mathrm{~h}$. The hydrolysate was then boiled for $15 \mathrm{~min}$ to inactivate the $\beta$-mannanase and centrifuged at $20,000 \times g$ for $15 \mathrm{~min}$ at $4^{\circ} \mathrm{C}$ to remove the insoluble fractions. Thereafter, the supernatants were measured by above mentioned of DNS colorimetric method.

\section{Phylogenetic analysis}

To reveal the evolutionary relationships of PcMan113 with other mannanases from various species, mannanase superfamily sequences were retrieved by blast search from a public database (http://www.ncbi.nlm.nih.gov/) and a phylogenetic tree was constructed using Molecular Evolutionary Genetics Analysis (MEGA) software (Hall. 2013).

\section{Structure Modeling of PcMan113}

Modeller 9.9.2. was used to construct a three-dimensional (3D) homology model of PcMan113 (Šali et al.1993). The crystal structure of mannanase from Bacillus sp. N16-5 (PDB ID: 7DV7, $1.4 \AA$ ) was chosen as the template. A sequence alignment between the template and PcMan113 was generated automatically using the align $2 \mathrm{~d}$ command, and the homology model was then generated via the automodel command. Each model was first optimized, and the structure was refined using simulated annealing in MD simulations (Abraham et al. 2015). The best model was chosen based on the values of the Modeller objective function and the DOPE assessment scores. The optimal model structure was visualized and analyzed using PyMOL software (http://www.pymol.org) (Humphrey et al. 1996).

\section{Site-directed mutagenesis}

To generate mannanase mutants with improved characteristics, site-directed mutagenesis was conducted with the PcMan113 expression plasmid as the template using the KOD-Plus-Mutagenesis kit (Toyobo, Japan). The mutations were confirmed by DNA sequencing. PcMan113 mutants were expressed and purified as described for the WT.

\section{Molecular dynamics simulations and calculations}


An external force was applied to the substrates center of mass along a predefined direction using steered molecular dynamics (SMD) simulations. The protein-substrate complex in Gromacs 5.1.2 software was created using the Gromos 96 53A6 force field. Subsequently, we obtained the mannobiose substrate parameters in the GROMOS96 53a6 force field from the Automated Topology Builder and Repository 2.0 webserver (https://atb.uq.edu.au/) (Koziara et al. 2014). The PcMan113-substrate complex and water molecules were placed into the simulation system, after which energy minimization was applied for the relaxation of the simulation system. After that, the constant-velocity SMD simulations were implemented, and the substrate center was pulled with a velocity of $0.01 \AA_{\mathrm{ps}^{-1}}$ and a spring constant of $1000 \mathrm{kcal}$ $\mathrm{mol}^{-1} \AA^{-2}$. The values of the PcMan113-WT and mutants were acquired by calculating an asymmetric distribution of sampling windows over the distance along the substrate binding channel.

\section{Statistical analysis}

All experiments were performed in triplicate. One-way analysis of variance (ANOVA) was used as a test for statistical significance, with a cutoff value of $p=0.05$.

\section{Results And Discussion}

\section{Sequence analysis and identification of PcMan113}

A novel endo-1.4- $\beta$-mannanases named as PcMan113 was found in a proprietary database derived from a Paenibacillus cineris. The PcMan 113 sequence was 987 bp, encoding a protein of 329 amino acid residues with a pl and molecular weights predicted to be 4.54 and $39.6 \mathrm{kDa}$, respectively. The protein showed 65\% similarity with a well characterized endo-1,4- $\beta$-mannanase from Bacillus sp. N16-5 (Liu et al. 2021). BLAST searches against the NCBI nonredundant and CAZy databases (Lombard et al. 2014), combined with conserved domain analysis, implied that PcMan113 belongs to the GH113 family. A phylogenetic tree was constructed based on the amino acid sequences of several similar proteins and representative $\beta$-mannanases form the $\mathrm{GH} 5, \mathrm{GH} 26$, and $\mathrm{GH} 113$ families using the neighbor-joining method, which further revealed that PcMan113 clustered with the GH113 like $\beta$-mannanase BaMan113A (Fig. S1).

PcMan113 was therefore identified as a novel member of GH family 113 with low sequence identity. The PcMan113 was predicted to be an intracellular enzyme according to SignalP analysis and it had no signal peptide. Sequence alignment with other identified GH113 family mannanases showed that several conserved residues (Fig. 1), and two catalytic residues (proton donor, E152; nucleophile, E232) and ten chemical substrate binding site residues (N98, W104, R105, E152, D183, Y204, E232, W282, D283, Y300) were strictly conserved among the GH113 family members.

\section{Expression, purification and identification of PcMan113}

PcMan113 was successfully overexpressed in E. coli BL21(DE3) and purified using Ni-NTA affinity chromatography (Fig. 2a). On size-exclusion chromatography, PcMan113 eluted as a single peak 
between the standard protein markers rabbit muscle actin $(15.7 \mathrm{kDa})$ and conalbumin $(43.0 \mathrm{kDa})$ (Fig. 2b), which indicated that purified PcMan113 was a monomer in solution. Based on these results and the SDS-PAGE, which revealed a single protein band, the mass of the purified protein was in good agreement with the predicted molecular weight of $39.6 \mathrm{kDa}$ (Fig. 2).

\section{Characterization of purified PcMan113}

Using LBG as the substrate, the PcMan113 exhibited an optimal activity in $\mathrm{Na}_{2} \mathrm{HPO}_{4}$-citrate buffer at pH 5.0 (Fig. 3a) and maintained more than $80 \%$ of its maximal activity at pH values between 4.5 and 7.0 . Moreover, the enzyme also showed considerable stability over an extensive pH range from 3.0 to 8.0, retaining over $60 \%$ of its initial enzyme activity after incubation without substrate at $37^{\circ} \mathrm{C}$ for $1 \mathrm{~h}$ (Fig. $3 \mathrm{~b}$ ).

The temperature-activity profile indicated that PcMan113 was a typical mesophilic hydrolase, exhibiting the highest activity at $55^{\circ} \mathrm{C}$ (Fig. $3 \mathrm{C}$ ) and thermostability at $45^{\circ} \mathrm{C}$ in $\mathrm{Na}_{2} \mathrm{HPO}_{4}$-citrate buffer. It retained more than $60 \%$ relative activity at temperatures ranging from 40 to $65^{\circ} \mathrm{C}$ (Fig. 3d). However, when incubated at $70^{\circ} \mathrm{C}$, the enzyme activity declined rapidly.

The effects of metal ions and the chelator EDTA on purified PcMan 113 were explored by incubating the enzyme at $55^{\circ} \mathrm{C}$ for $1.0 \mathrm{~h}$. The results showed that $5 \mathrm{mM} \mathrm{Zn}^{2+}$ could slightly enhance the enzyme activity to $115 \%$, while $\mathrm{Cu}^{2+}$ and $\mathrm{Ag}^{+}$greatly inhibited the enzyme activity. Moreover, $\mathrm{Mn}^{2+}$ and $\mathrm{Fe}^{2+}$ reduced the enzyme activity by approximately $10 \%$, and EDTA also moderately inhibited its activity (Fig. S2).

\section{Substrate selectivity and kinetic properties of PcMan113}

To determine the substrate selectivity of PcMan113, we incubated purified PcMan113 with various substrates including LBG, KG, GG, sodium carboxymethyl cellulose (SCC), beechwood xylan (BX), mannobiose (M2), mannotriose (M3), mannotetraose (M4), mannopentaose (M5) and mannohexaose (M6). The purified PcMan113 showed maximal activity towards M5 $\left(8.2 \times 10^{3} \mathrm{U} / \mathrm{mg}\right)$, followed by M4 $\left(7.5 \times 10^{3} \mathrm{U} / \mathrm{mg}\right), \mathrm{M} 6\left(6.9 \times 10^{3} \mathrm{U} / \mathrm{mg}\right), \mathrm{M} 3\left(3.2 \times 10^{3} \mathrm{U} / \mathrm{mg}\right)$ and $\mathrm{M} 2\left(2.6 \times 10^{2} \mathrm{U} / \mathrm{mg}\right)$. The purified PcMan113 showed much lower activity towards KG $\left(68.2 \times 10^{2} \mathrm{U} / \mathrm{mg}\right), \mathrm{LBG}(31.7 \mathrm{U} / \mathrm{mg})$ and GG (2.1 $\mathrm{U} / \mathrm{mg}$ ) orderly (Table1), and no activity was observed with SCC or BX. The specific activity of PcMan 113 toward KG was higher than toward LBG or GG, which was consistent with other enzymes from the GH 113 family, such as BaMan113A from Bacillus sp. N16-5 (Liu et al. 2021), as well as AaManA (David et al. 2018) and Man113A from Alicyclobacillus sp. strain A4 (You et al., 2018). By contrast, mannanases from GH families of 134, 26 and 5, such as AoMan134A (Hogg et al. 2003), CrMan26 (Mandelli et al. 2020), and RmMan5A (Song et al. 2018), exhibit a high preference for LBG. PcMan113 and other GH family mannanases have different preferences toward LBG and KG, which are related to structural differences in space for accommodating the substrate with galactose side chains in a non-catalytic binding mode (Jin et al. 2016; Kumagai et al. 2015; Le et al. 2005).

The kinetic properties of purified PcMan113 were analyzed using mannooligosaccharides as substrates, including mannobiose (M2), mannotriose (M3), mannotetraose (M4), mannopentaose (M5) and 
mannohexaose (M6). The kinetic parameters of PcMan113 for these substrates were calculated by nonlinear fitting to the Michaelis-Menten equation (Table 2) (Kumagai et al. 2015) The $K_{m}$ values for M2, M3, M4, M5 and M6 were 66.1, 35.4, 5.1, 4.7 and $8.2 \mathrm{mM}$, respectively, indicating that PcMan113 has the highest affinity for M5, followed by M4 and M6. Moreover, PcMan113 showed higher catalytic efficiency with M5 $\left(6.55 \mathrm{mM}^{-1} \mathrm{~s}^{-1}\right)$ and M4 $\left(5.19 \mathrm{mM}^{-1} \mathrm{~s}^{-1}\right)$ than with $\mathrm{M} 6$, and the lowest catalytic efficiency with M2 $\left(0.035 \mathrm{mM}^{-1} \mathrm{~s}^{-1}\right)$.

It was reported that $\mathrm{GH} 113$ family members showed considerable transglycosylation activity and could hydrolyze mannooligosaccharides with degrees of polymerization (DP) above 3 (Zhang et al. 2008; Xia et al. 2016). Our results indicated that the smallest substrate of PcMan113 was M3 (Table 1 and 2), which was consistent with the catalytic properties of other $\mathrm{GH} 113$ family members. Compared to other endo- $\beta$ mannanases, PcMan113 showed poor catalytic efficiency on M2 $\left(0.035 \mathrm{mM}^{-1} \mathrm{~s}^{-1}\right)$ and M3 $(0.20$ $\left.\mathrm{mM}^{-1} \mathrm{~s}^{-1}\right)$, and the catalytic efficiency toward M3 much lower than that of Man113A (6.54 $\left.\mathrm{mM}^{-1} \mathrm{~s}^{-1}\right)$ (Xia et al. 2016) and PaMan5A (26.67 $\mathrm{mM}^{-1} \mathrm{~s}^{-1}$ ) (Couturier et al. 2013). The enzyme hydrolyzed M2 slowly (data not shown), suggesting that the hydrolysis products of PcMan113 would be mainly M2 and mannose, which makes it suitable to degrade mannans.

\section{Structural homology analysis and active site screening of PcMan113 protein}

A structural homology model of PcMan113 was generated using BaMan113A (PDB ID: 7DV7) as the template. The Ramachandran plot indicated that $97 \%$ of the residues were in the favored and allowed regions, indicating that the model of PcMan113 is plausible. PcMan113 possesses two polypeptide chains in an asymmetric unit, each folding into a typical ( $\beta / a)$ 8TIM-barrel architecture (Fig. 4a and Fig. S3), which is consistent with the typical features of GH113 family enzymes (You et al., 2018). The catalytic residues E152 and E232 are located at the center of the $\beta$-barrel, which forms a hydrophobic area near which a deep cavity is observed. The PcMan113 may have a semi-enclosed substrate-binding pocket cleft, which was occupied by amino acids residues such as N98, W104, R105, F110, D183, Y204, W282, D283, Y300, which formed the substrate binding site of PcMan113 (Figs. 4b and S4).

Thus, this cavity accommodates the substrate and these residues interact with the substrate. The intermolecular interface involves four regions, T58-H60, F110-E118, V154-Q155, and K184-Q186, which are located in loops (Fig. S5). Among them, loop F110-E118 might act as a lid that seals the active site, which has an effect on the entrance of substrates and release of products.

\section{Rational engineering of PcMan113 enzyme}

Based on the discussed structural features of PcMan113 enzyme, a single point mutant library containing 23 single mutants at 9 residues was constructed and evaluated for activity towards the industrial substrates KG and LBG (Fig. 5a). Mutation of residues W20, W104, E118, P119, K184, W248 and W284 reduced the enzyme activity, indicating these residues played important roles in substrate 
binding. By contrast, mutation of residues F110 and N246 enhanced activity towards substrates of KG and LBG, and these two residues were selected as targets for site-directed mutagenesis.

On the basis of substrate-binding pocket analysis, the six mutants F110A, F110E, N246A, N246F, N246W and N246Y were constructed and evaluated. Among them, the four mutants F110E and N246F/W/Y showed higher catalytic activity towards KG and LBG than wild-type PcMan113 (WT) (Fig. 5a). The mutant F110E (PCMT1) showed respective 2.0- and 2.2-fold increases of catalytic activity toward KG and LBG compared with the WT. PCMT1 showed high substrate affinity and catalytic efficiency with a $K_{\mathrm{m}}$ of $1.40 \mathrm{mM}$ and a $k_{\text {cat }}$ of $35.20 \mathrm{~s}^{-1}$, respectively (Table S1). The biochemical data revealed that the F110E118 loop may affects enzymatic hydrolysis by interacting with F110 and E118 from the adjacent monomers. In addition, the loop F110-E118 was proposed to interact with the substrate and active sites, and F110 was mutated to the acidic residue glutamate or nonpolar residues with small side chains, which may affect substrate recognition and enzymatic hydrolysis.

Mutating N246 to aromatic residues led to a slight reduction in enzyme activity (Fig. 5a), and some studies reported that Trp-mediated binding plays an important role in $\beta$-mannanses of GH113 (Kumagai et al. 2015; Xia et al. 2016; Liu et al. 2021), suggesting that the stacking interaction at this position is conducive to improving enzyme activity. Therefore, N246 was replaced with Phe, Trp and Tyr in three different mutants. The most remarkable results were observed for N236Y (PcMT2), which exhibited 233\% and $246 \%$ relative activity with KG and LBG, respectively (Fig. 5a). The enzyme kinetic parameters of PcMT2 were significantly enhanced, which much higher substrate affinity and catalytic efficiency on M5 $\left(K_{\mathrm{m}}, 1.8 \mathrm{mM} ; k_{\text {cat }} / K_{\mathrm{m}}, 19.30\right)$ compared with the WT (Table S1). These results were in agreement with previous reports that improving stacking interactions between the substrate and protein at the position of N246 could significantly enhance the catalytic activity (Liu et al. 2021; You et al. 2018; Xia et al. 2016).

Furthermore, the mutations with improved activity on KG and LBG were combined, resulting in three additional double mutants of PcMan113 (F110E/N246F, F110E/N246Y and F110E/N246W). The enzyme activity measurements reveled that all double mutants had much higher hydrolysis activity towards LBG and KG than the WT and the corresponding single point mutants (Fig. 5b). The F110E/N246Y mutant (PcMT3) not only exhibited a great improvement in hydrolytic activity (4.6- and 3.5-fold respective increases with KG and LBG), but also showed higher substrate affinity and catalytic efficiency $\left(K_{\mathrm{m}}, 0.9\right.$ $\left.\mathrm{mM} ; k_{\text {cat }} / K_{\mathrm{m}}, 37.44\right)$ with M5 than the WT enzyme (Fig. 5b and Table S1). However, these results were different from similar mutants of BaMan113A, which exhibited reduced substrate affinity and catalytic efficiency [35]. Thus, PcMT1, PcMT2 and PcMT3 had clearly increased activity in the hydrolysis of mannans and manno-oligosaccharides, which indicated that the substrate preference of the enzyme has not been altered. At the same time, these data also demonstrated that PcMan113 hydrolyzed substrates in a standard endo-acting mode, indicating that PcMan113 can degrade mannans more completely, which was similar to the reported enzyme Man113A (Xia et al. 2016).

\section{MD simulations and structural analysis of the PcMT3 variant}

Page $10 / 21$ 
To further explore the differences between the WT enzyme and improved variant PcMT3 in catalytic activity and protein-substrate interactions, mannobiose was docked into the binding sites of WT and PcMT3 in 30 ns MD simulations. The results indicated that the binding energy of PcMT3 with mannobiose was dramatically increased compared with the WT $(-7.85 \mathrm{kcal} / \mathrm{mol} \mathrm{vs} .-37.83 \mathrm{kcal} / \mathrm{mol})$ (Table S2). The root mean-square deviations (RMSD) over $30 \mathrm{~ns}$ were calculated to investigate the stability of the WT/PcMT3-mannobiose complexes (Fig. S6a). PcMan113-WT reached the equilibrium state after $15 \mathrm{~ns}$ of simulation, while Pc-MT3 was stable state after $10 \mathrm{~ns}$ of simulation. The average RMSD values of variants PcMT1/2/3 were $0.26 \pm 0.06 \mathrm{~nm}, 0.30 \pm 0.06 \mathrm{~nm}$ and $0.25 \pm 0.04 \mathrm{~nm}$, respectively, all of which were lower than that of the Pc-WT complex $(0.32 \pm 0.08 \mathrm{~nm})$, indicating that all variants were more stable.

Moreover, the root mean square fluctuations (RMSF) were calculated to assess the mobility of protein residues, and the results showed that both the WT and the variants had low fluctuations ranging from 0.2 to $0.3 \mathrm{~nm}$ (Fig. S6b). Compared with the WT, some residues in loops that are part of the substrate binding site (such as F110 and N246) of PcMT3 had higher RMSF values, reaching $>0.4 \mathrm{~nm}$ in loop R243-N255, and $>0.3 \mathrm{~nm}$ in loop F110-E118, indicating greater flexibility in these loops than in the WT (Fig. S6b). The radius of gyration $(\mathrm{Rg})$ values are an indicator of the reliability of protein homology modeling, and the $\mathrm{Rg}$ values of WT-PcMan113 and variants all reached between 1.9 and $1.95 \mathrm{~nm}$ (Fig. S7), suggesting that the homology modeling was reliable.

In the WT-mannobiose complex, a narrow substrate access channel to the active site was observed (Fig. 6a). By contrast, the mutations in PcMT3 led to a somewhat bigger space in the substrate binding channel and the substrate could easily enter the catalytic center (Figs. 6b, 6d and 7). Based on the previous structural analysis of the homology model and the N246Y-mediated structural effects on the substrate-binding tunnel, we hypothesized that N246 may play a pivotal role in blocking the access of the substrate into the active site, while the newly introduced $\mathrm{Y} 246$ side chain adds a stacking interaction for substrate binding. The N246Y mutation possibly removed steric hindrance to widen the available substrate-binding space inside the binding pocket (Fig. 6a-d). Indeed, the N246Y mutant exhibited a $46 \%$ activity improvement with LBG compared with the WT, which was also in agreement with the MD simulations (Fig. S6 and Table S2). When F110 was mutated to Glu in PcMT3, its enzyme activity was further enhanced, and its RMSD indicated that the F110-E118 loop had greater flexibility (Fig. S6b). We therefore hypothesized that loop F110-E118 possibly interacted with the substrate and active site (Figs. S5 and 7). In addition, when site of F110 was mutated to E, E110 was found has a small side chain, which may affect substrate recognition and allow easy entrance into the binding pocket.

Furthermore, we also observed an interesting conformational change in residue W248, which was altered upon ligand binding (Fig. 7). This resulted in a more conducive conformation of the mannobiose binding channel, bringing the residue W248 that was proposed to act as a lid of the substrate-binding channel in close proximity to the substrate in the active site.

\section{Conclusion}


The novel GH113 family endo- $\beta$-mannanase PcMan113 from Paenibacillus cineris was cloned, expressed, purified and characterized. Recombinant PcMan113 showed a broader substrate spectrum and better characteristics than previously reported enzymes. It exhibited the highest enzyme activity at pH 5.0 and $55^{\circ} \mathrm{C}$. It was acid tolerant, with a pH range of $3.0-8.0$, and thermostable under $45^{\circ} \mathrm{C}$. Homology modeling and structural analysis of PcMan113 revealed key active site residues. Finally, we obtained the highly active mutant PcMT3 (F110E/N246Y), which not only exhibited a great improvement in hydrolytic enzyme activity (4.6- and 3.5-fold increases with KG and LBG), but also showed higher substrate affinity and catalytic efficiency $\left(K_{\mathrm{m}}, 0.9 \mathrm{mM} ; k_{\text {cat }} / K_{\mathrm{m}}, 37.44\right)$ with M5 than the WT enzyme. Moreover, molecular dynamics (MD) simulations revealed that the binding energy of PcMT3 with mannobiose was dramatically increased compared with that of the WT $(-7.85 \mathrm{kcal} / \mathrm{mol} \mathrm{vs} .-37.83 \mathrm{kcal} / \mathrm{mol})$, indicating that a decrease of steric hindrance resulted in increased binding energy. A notable conformational change of residue W248 enlarged the substrate binding channel. Our work on PcMan113 not only enriches the available GH113 family enzymes for structure-based engineering, but also provides the significantly improved variant PCMT3 for potential commercial use in the food and feed industries.

\section{Abbreviations}

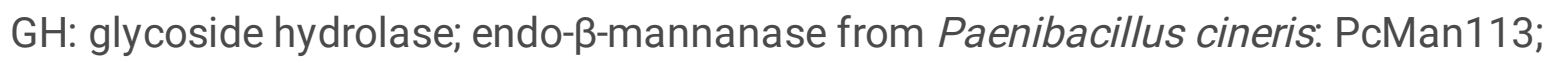

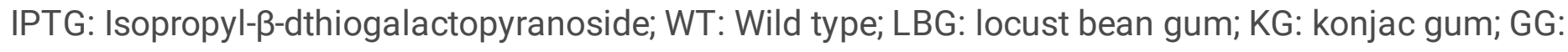
guar gum; SCC: sodium carboxymethyl cellulose; BX: beechwood xylan; M2: mannobiose; M3: mannotriose; M4: mannotetraose, M5: mannopentaose; M6: mannohexaose; MD: Molecular dynamics; RMSF: Root-mean-square fuctuations; PME: Particle mesh Ewald; RMSD: The root mean-square deviations; Rg: The radius of gyration values.

\section{Declarations}

\section{Acknowledgements}

The Project Supported by the Foundation (No. KF201921 and ZZ20200131) of State Key Laboratory of Biobased Material and Green Papermaking, Qilu University of Technology, Shandong Academy of Sciences.

\section{Authors' contributions}

Hui-Min Qin, Zhixiong Zeng and Teng-Fei Wang designed the research. Dengyue Sun, Li Chao, Pengpeng Cui, Jie Zhang performed the experiments. Yaolin Zhou and Mian Wu performed the molecular modeling and molecular dynamics simulations. Chao Li, Dengyue Sun and Xia Li Analyzed the data. Hui-Min Qin and Dengyue Sun Wrote the paper. Zhixiong Zeng and Hui-Min Qin have approved the final version of the manuscript. All authors read and approved the final manuscript.

\section{Funding}


The Project Supported by the Foundation (No. KF201921 and ZZ20200131) of State Key Laboratory of Biobased Material and Green Papermaking, Qilu University of Technology, Shandong Academy of Sciences.

\section{Availability of data and materials}

The data which this article is based upon are all included in this manuscript and the additional files associated with it.

\section{Ethics approval and consent to participate}

Not applicable.

\section{Consent for publication}

Not applicable

\section{Competing interests}

The authors declare no competing financial interests.

\section{References}

1. Abraham M, Murtola T, Schulz R, Páll S, Smith J, Hess B, Lindahl E (2015) GROMACS: High performance molecular simulations through multi-level parallelism from laptops to supercomputers. SoftwareX 1-2:19-25

2. Akino T, Nakamura N, Horikoshi K (1988) Characterization of three BETA-mannanases of an alkalophilic Bacillus sp. Agric Biol Chem 52:773-779

3. Cao LL, Liu WB, Wang LJ (2018) Developing a green and edible film from cassia gum: The effects of glycerol and sorbitol. J CLEAN PROD 175:276-282

4. Couturier M, Roussel A, Rosengren A, Leone P, Stålbrand H, Berrin JG (2013) Structural and biochemical analyses of glycoside hydrolase families 5 and $26 \beta$-(1,4)-mannanases from Podospora anserine reveal differences upon manno-oligosaccharide catalysis. J Biol Chem 288:14624-14635

5. David A, Chauhan PS, Kumar A, Angural S, Kumar D, Puri N, Gupta N (2018) Coproduction of protease and mannanase from Bacillus nealsonii PN-11 in solid state fermentation and their combined application as detergent additives. Int J Biol Macromol 108:1176-1184

6. Eom GT, Oh JY, Park JH, Jegal J, Song JK (2016) Secretory production of enzymatically active endo$\beta-1,4-m a n n a n a s e$ from Bacillus subtilis by ABC exporter in Escherichia coli. Process Biochem 51(8):999-1005

7. Ghasemian M, Jahanian R (2016) Dietary mannan-oligosaccharides supplementation could affect performance, immunocompetence, serum lipid metabolites, intestinal bacterial populations, and ileal nutrient digestibility in aged laying hens. Anim Feed Sci Technol 213:81-89 
8. Giannenas I, Doukas D, Karamoutsios A, Tzora A, Bonos E, Skoufos I, Tsinas A, Christaki E, Tontis D, Florou-Paneri P (2016) Effects of Enterococcus faecium, mannan oligosaccharide, benzoic acid and their mixture on growth performance, intestinal microbiota, intestinal morphology and blood lymphocyte subpopulations of fattening pigs. Anim Feed Sci Technol 220:159-167

9. Hall BG (2013) Building phylogenetic trees from molecular data with MEGA. Mol Biol Evol 30(5):1229-1235

10. Hogg D, Pell G, Dupree P, Goubet F (2003) The modular architecture of Cellvibrio japonicus mannanases in glycoside hydrolase families 5 and 26 points to differences in their role in mannan degradation. Biochem J 371:1027-1043

11. Humphrey W, Dalke A, Schulten K (1996) VMD-Visual Molecular Dynamics. J Mol Graphics 14:33-38

12. Jin Y, Petricevic M, John A, Raich L, Jenkins H, Portela De Souza L, Cuskin F, Gilbert HJ, Rovira C, Goddard-Borger ED, Williams SJ, Davies GJ (2016) A $\beta-1,4$-mannanase with a lysozyme-like fold and a novel molecular catalytic mechanism. ACS Cent Sci 2:896-903

13. Koziara KB, Stroet M, Malde AK, Mark AE (2014) Testing and validation of the Automated Topology Builder (ATB) version 2.0: prediction of hydration free enthalpies. J Comput Aided Mol Des 28:221233

14. Kumagai Y, Yamashita K, Tagami T, Uraji M, Wan K, Okuyama M, Yao M, Kimura A, Hatanaka T (2015) The loop structure of Actinomyceteglycoside hydrolase family 5 mannanases governs substrate recognition. FEBS J 282:4001-4014

15. La Rosa SL, Leth ML, Michalak L, Hansen ME, Pudlo NA, Glowacki R, Westereng B (2019) The human gut Firmicutev Roseburia intestinalisis a primary degrader of dietary $\beta$-mannans. Nat Commun 10:114

16. Le Nours J, Anderson L, Stoll, Stålbrand Lo, Leggio L (2005) The structure and characterization of a modular endo- $\beta$-1,4-mannanase from Cellulomonas fimi. Biochemistry 44:12700-11270

17. Li YX, Yi P, Liu J, Yan QJ, Jiang ZQ (2017) High-level expression of an engineered B-mannanase (mRmMan5A) in Pichia pastoris for manno-oligosaccharide production using steam explosion pretreated palm kernel cake. Bioresour Technol 256:30-37

18. Liu W, Ma C, Liu W, Zheng Y, Chen C-C, Liang A, Luo G, Li, Ma J, Song Y, Guo R-T, Zhang T (2021) Functional and structural investigation of a novel $\beta$-mannanase baman113a from bacillus sp. N16-5. Int J Biol Macromol 182:899-909

19. Lombard V, Golaconda Ramulu H, Drula E, Coutinho PM, Henrissat B (2014) The carbohydrate-active enzymes database (CAZy) in 2013. Nucleic Acids Res 42:D490-D495

20. Lowry OH, Rosebrough NJ, Farr AL, Randall RJ (1951) Protein measurement with the folin-phenol reagent. J Biol Chem 193:265-275

21. Malgas S, van Dyk JS, Pletschke BI (2015) A review of the enzymatic hydrolysis of mannans and synergistic interactions between $\beta$-mannanase, $\beta$-mannosidase and $\mathrm{a}$-galactosidase. World $\mathrm{J}$ Microbiol Biotechnol 31:1167-1175 
22. Mandelli F, de Morais MAB, de Lima EA, Oliveira, Persinoti GF, Murakami MT (2020) Spatially Remote Motifs Cooperatively Affect Substrate Preference of a Ruminal GH26-type Endo- $\beta$-1,4-Mannanase. J Biol Chem 295:5012-5021

23. Mano MC, Neri-Numa IA, Da Silva JB, Paulino BN, Pessoa MG, Pastore GM (2018) Oligosaccharide biotechnology: An approach of prebiotic revolution on the industry. Appl Microbiol Biot 102:17-37

24. Moreira LR, Filho EX (2008) An overview of mannan structure and mannan-degrading enzyme systems. Appl Microbiol Biot 79:165-178

25. Pérez-Burillo S, Pastoriza S, Fernández-Arteaga A, Luzón G, Jiménez-Hernández N, D’Auria G, RufiánHenares J (2019) Spent coffee grounds extract, rich in mannooligosaccharides, promotes a healthier gut microbial community in a dose-dependent manner. J Agric Food Chem 67:2500-2509

26. Pongsapipatana N, Damrongteerapap P, Chantorn S, Sintuprapa W, Keawsompong S, Nitisinprasert S (2016) Molecular cloning of kman coding for mannanase from Klebsiella oxytoca KUB-CW2-3 and its hybrid mannanase characters. Enzym Microb Technol 89:39-51

27. Rai PR, Tiwary AK, Rana V (2012) Superior disintegrating properties of calcium cross-linked cassia fistula gum derivatives for fast dissolving tablets. Carbohyd Polym 87:1098-1104

28. Saha BC (2003) Hemicellulose bioconversion. J Ind Microbiol Biot 30:279-291

29. Šali A, Blundell T (1993) Comparative protein modelling by satisfaction of spatial restraints. J Mol Biol 234:779-815

30. Shimizu M, Kaneko Y, Ishihara S, Mochizuki M, Sakai K, Yamada M, Murata S, Itoh E, Yamamoto T, Sugimura $Y(2015)$ Novel $\beta$-1,4-mannanase belonging to a new glycoside hydrolase family in Aspergillus nidulans. J Biol Chem 290:27914-22792

31. Song YJ, Sun WY, Fan Y, Xue YF (2018) Galactomannan degrading enzymes from the mannan utilization gene cluster of alkaliphilic Bacillus sp. N16-5 and their synergy on galactomannan degradation. J Agric Food Chem 66:11055-11063

32. Srivastava M, Kapoor VP (2005) Seed Galactomannans: An Overview. Chem Biodiversity 2:295-317

33. Srivastava PK, Kapoor M (2017) Production, properties and applications of endo- $\beta-1,4-m a n n a n a s e s$. Biotechnol Adv 35:1-19

34. Srivastava PK, Kapoor M (2014) Cost-effective endo-mannanase from Bacillussp. CFR1601 and its application in generation of oligosaccharides from guar gum and as detergent additive. Prep Biochem Biotechnol 44:392-417

35. Srivastava PK, Kapoor M (2017) Production, properties, and applications of endo- $\beta$-mannanases. Biotechnol Adv 35:1-9

36. Torrecillas S, Montero D, Izquierdo M (2014) Improved health and growth offish fed mannanoligosaccharides: potential mode of action. Fish Shellfish Immunol 36:525-544

37. Xia W, Lu H, Xia M, Cui Y, Bai Y, Qian L, Shi P, Luo H, Yao B (2016) A novel glycoside hydrolase family 113 endo- $\beta-1,4-m a n n a n a s e$ from Alicyclobacillus sp. strain A4 and insight into the substrate recognition and catalytic mechanism of this family. Appl Environ Microbiol 82:2718-2727 
38. Yamabhai M, Sak-Ubol S, Srila W, Haltrich D (2016) Mannan biotechnology: from biofuels to health. Crit Rev Biotechnol 36:32-42

39. Yang H, Shi PJ, Lu HQ, Wang HM, Luo HY, Huang HQ, Yang PL, Yao B (2015) A thermophilic $\beta$ mannanase from Neosartorya fischeri 1 with broad pH stability and significant hydrolysis ability of various mannan polymers. Food Chem 173:283-289

40. Yang J, Chen Q, Zhou B, Wang X, Liu S (2019) Mannooligosaccharide preparation by the hydrolysis of konjacflour with a thermostable endo mannanase from Talaromyces cellulolyticus. J Appl Microbiol 127:520-532

41. You X, Qin Z, Yan QJ, Yang SQ, Li YX, Jiang ZQ (2018) Structural insights into the catalytic mechanism of a novel glycoside hydrolase family 113 beta-1,4-mannanase from Amphibacillus xylanus. J Biol Chem 293:11746-11757

42. Zhang YL, Ju JS, Peng H, Gao F, Zhou C, Zeng Y, Xue YF, Li Y, Henrissat B, Gao GF, Ma YH (2008) Biochemical and structural characterization of the intracellular mannanase AaManA of Alicyclobacillus acidocaldarius reveals a novel glycoside hydrolase family belonging to clan $\mathrm{GH}-\mathrm{A}$. J Biol Chem 283:31551-31558

43. Zhao D, Zhang X, Cui DZ, Zhao M (2012) Characterisation of a novel white laccase from the deuteromycete fungus Myrothecium verrucaria NF-05 and its decolourisation of dyes. PLoS One 7:e38817

44. Zheng C, Li F, Hao Z, Liu T (2018) Effects of adding mannan oligosaccharides on digestibility and metabolism of nutrients, ruminal fermentation parameters, immunity, and antioxidant capacity of sheep. J Anim Sci 96:284-292

\section{Figures}
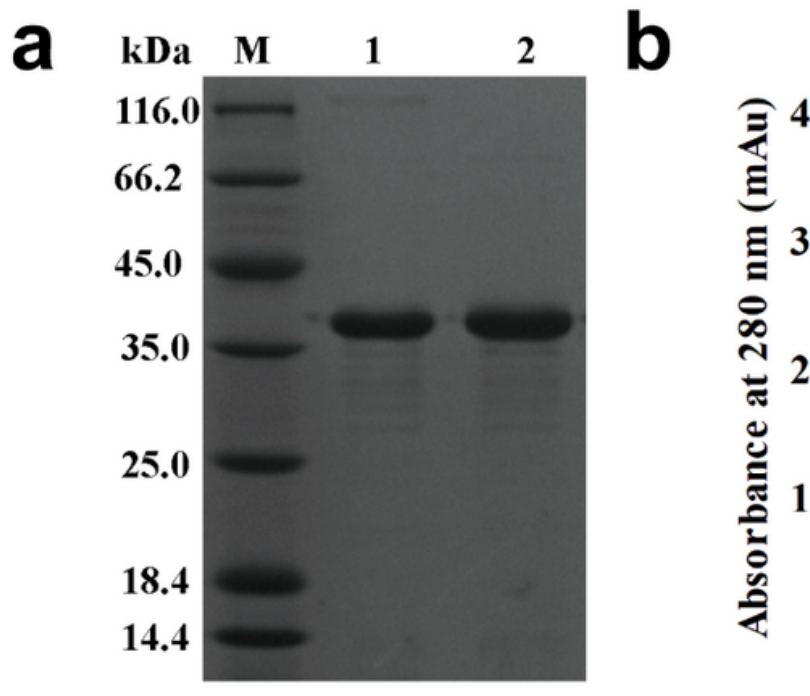

Figure 1 
Purification of ManAC by SDS-PAGE (a) and size-exclusion chromatography analysis of the recombinant PcMan113 (b). Lane M: marker; Lane 1-2: The PcMan purified by size-exclusion chromatography.

$4 C D 8$

AaManA_4CD8 AxMan113A_5YLH

PcMam113

BaMan113A_7dv7 $\alpha 1$

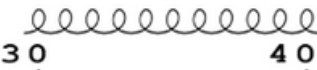

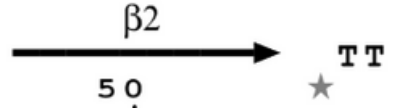<smiles>CCC</smiles>

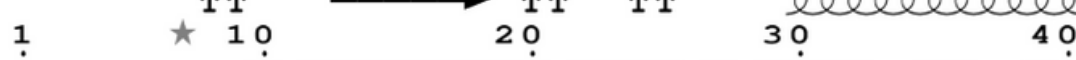
MGR I E A D I G IRGMTFGFV GQHGTWGTDEARASMRA LA. EQP F NWVT LAFAGLMEHPG

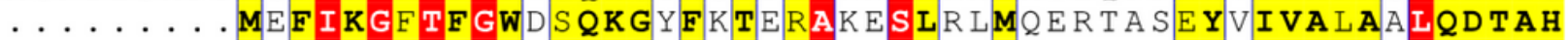
MN LMRNNLLEGYIGGMTWGWTG IRGTWNTPEAGHSMEEMVKRLGVNWTAVT FAALQD TAH

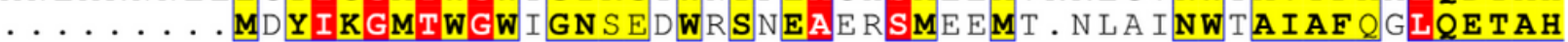

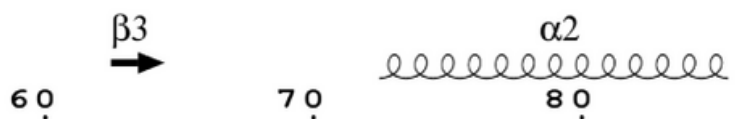

AaManA_4CD8 AXMan113A_5YLH PcMam113 BaMan113A_7dv7

DP A I A Y P P V TVSDDE I A MAE LAHALGLKVCLKPTVNCRDGTWRGEIREEKE H GPDES STEVDFQGSH MVDDDELIELIDYAKSLGLKVILKPTVNCRNGTWRAHINF F D MDIPGEPT S T E I H F A E E P T VTDE EVRHA I A AKRLGLRVCLKPVVNCT DG T WRAHINFF D D VPCE P K SPDITEA E P P MVTDENVRWA I A KAKSLGLSVILKP IVNVRDG TWRAHINFF D KEVPCEP T

$\alpha 3$

120 elebelebelebelel

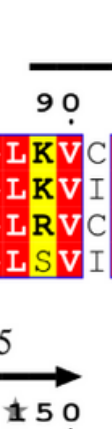

$\Lambda$

$\alpha 4$

elebelecelee 170

AaManA_4CD8 AxMan113A_5YLH PcMam113 WE A WFGSYS D MMAHYAHVAKRTGCEMFCVGCEMT TAEPHEAMWRETIARVRTEYDGLVTY BaMan113A_7dv7

\section{WDEWFESY INY $Q K H Y A K I A E K T N C E M F V V G C E M V Q A E R R E D K W R E I I A E V R K D Y R G L V T Y$} WS EWFDSYTRFMLHYAAMAEETGCEMLCIGCEMVQT DRREREWRELIRQVRQVYSGIVTY WS QWFKSYES FMLHYAKLAEDTGCEMLCIGCEMVQTERREKEWRDLIQKVRQVYSGI ITY
$\rightarrow$
180
$\eta 2$
190
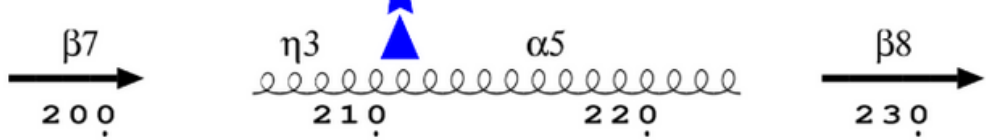

AaManA_4CD8 AxMan113A_5YLH PcMam113 BaMan113A_7dv7

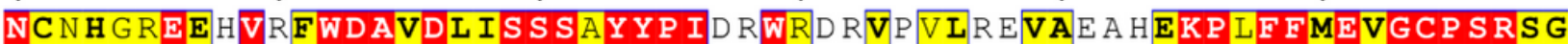
NT DKYQEDNVKFWDALDVISSSGYYPINDWDRQLDRIEAVVKQYDKPFFFVEAGCPSRSG NCDKY QEG QVAWWD AVDVISS SGYYPID SWEE QLDRIEPVVRRHDKPFF FMEAGCPSRTG NCDKYQEDEVTWWDAVDVMS S SGYYP I G SWEH HESRIKKIVESWQKPFF FMEAGCP SRLE

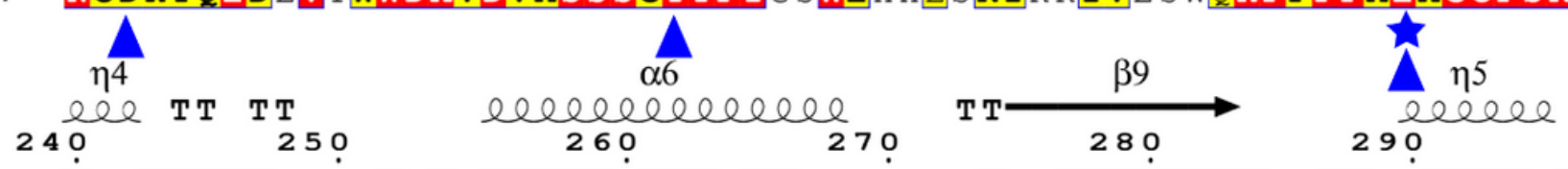

AaManA_4CD8 AXMan113A_5YLH PcMam113 BaMan113A_7dv7

SGA CPWDYRHP . GAVC LDEQAR FYEAMFA AMP DEP WF KGYMLWEWPWK LYPREAASEDGS SA LIP NKWDLE. GA IN L QE QADYY QVMFEKT A S R WVGGF GLWDWQTYLYDEKDATKNDD SSRIPNDWGLP. GD P NEDEQAAYYERMFEACASRSWVGGFMLWDWPA ALYAPEDAAGNDD SG S VPNDWNKNRGQI D MDQRVFYE EMFKF F H G KWF YGF MLWDWPA KLYR LEDASENDD

$$
\text { 3о0 beleelecel } 320
$$

AaManA_4CD8 AxMan113A_5YLH PcMam113

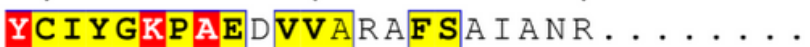
YGVFGKPAERVIKAYYQSR............... YCP YAKKAEGIIRD HYTA $Q$ I RELGGLPAQP YCVYGKPAAEVIKS F F TSNKIAKR......

BaMan113A_7dv7

Figure 2

Mutiple sequence alignment of PcMan113 with other reported $\beta$-1,4-mannanase belonged to $\mathrm{GH}$ family 113 members (AaManA_4CDB, AxMan113A_5YLH and BaMan113A). Residues forming the secondary structures of PcMan113 are highlighted above the sequences. Identical residues are shown in white on red background. The two catalytic residues (E152 and E232) and other eight proposed substrate binding 
sites (N98, W104, R105, D183, Y204, W282, D283, Y300) are marked by blue stars and triangle, respectively.

a

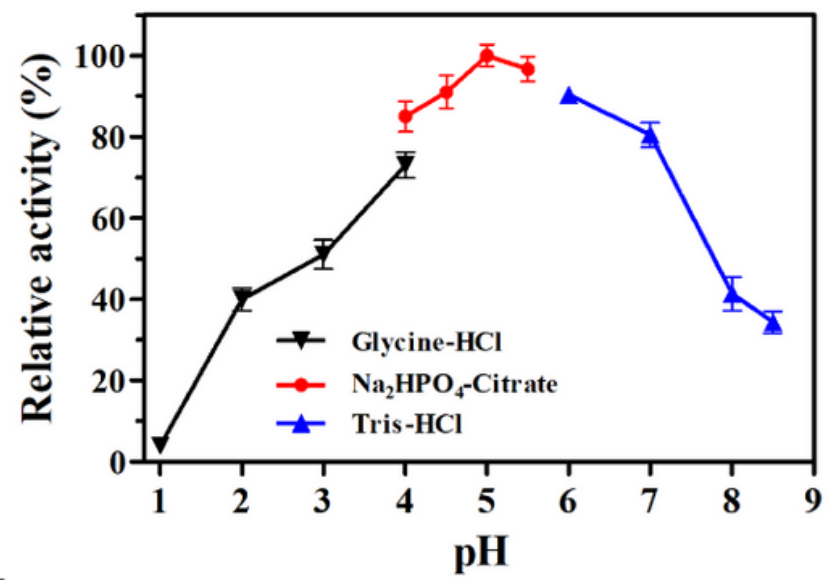

b

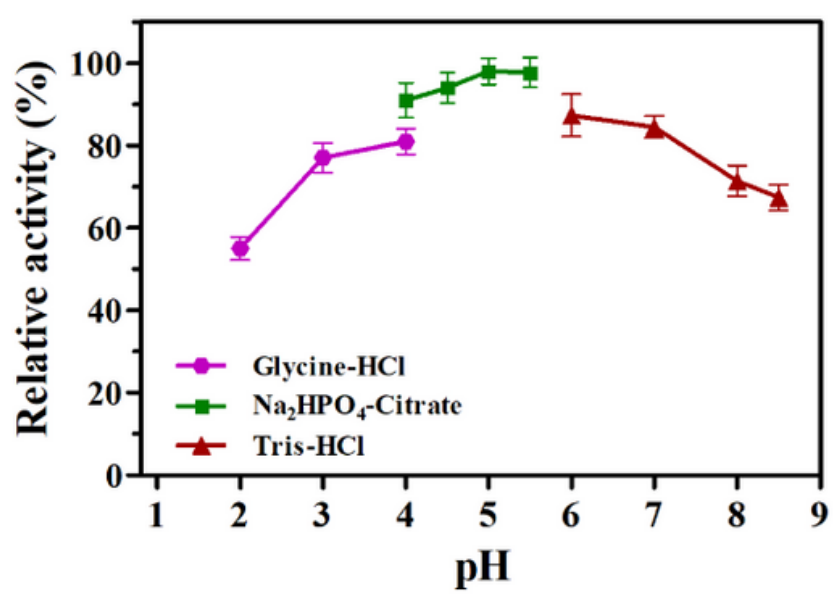

C

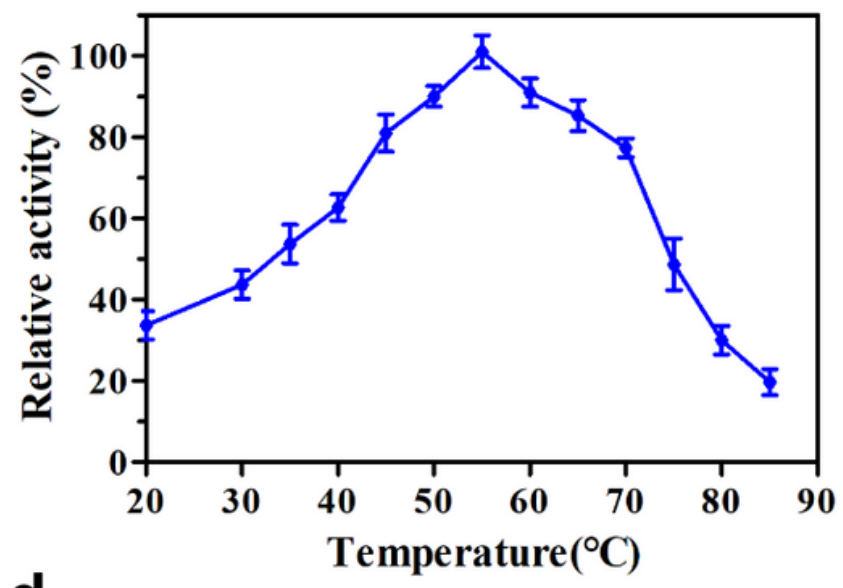

d

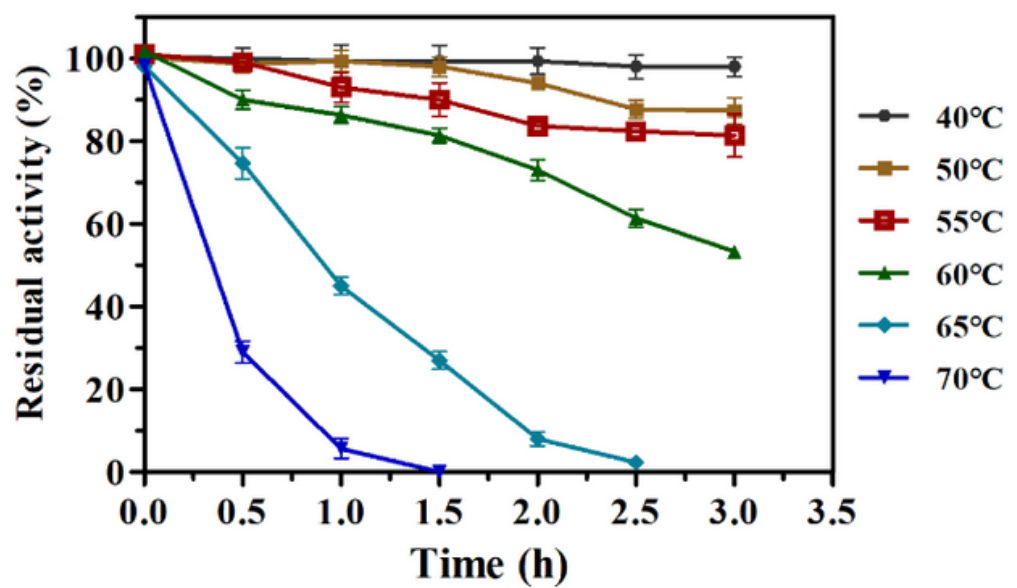

Figure 3

Determination of PcMan113 optimum characters' optimal pH (a) and pH stability (b), temperature (c) and thermal stability (d).
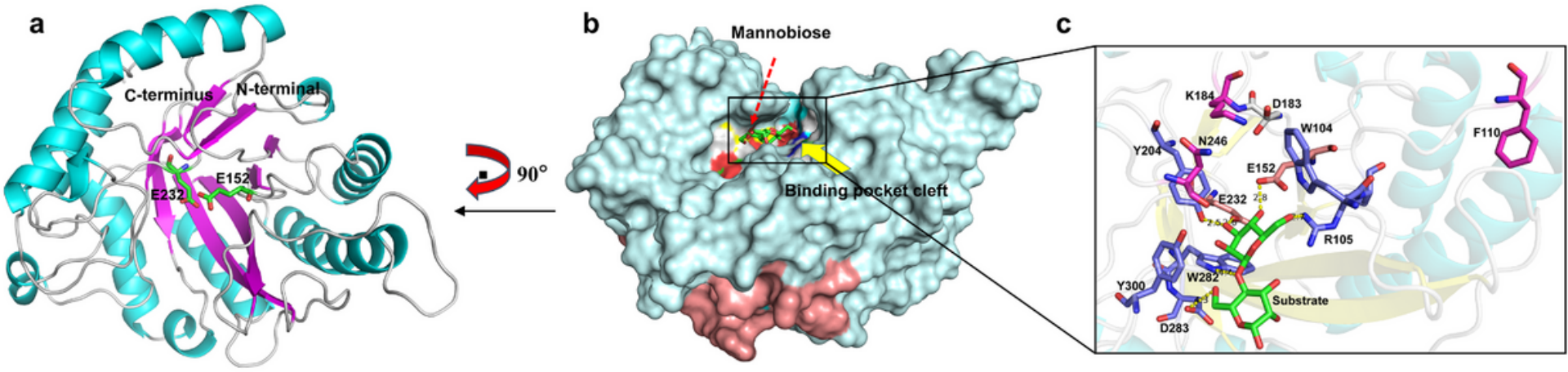

Figure 4

Structural homology analysis of the PcMan113-substrate complex. (a) Cartoon representation of the overall structure of PcMan113. Helices are marked by color cyan, $\beta$-sheets in magenta, and loops in white 
(b) Surface representation of the overall structure of PcMan113-mannobiose complex. (c) Enlarged insight of the catalytically substrate binding pocket sites. The two catalytic residues are marked as orange sticks. The two residues are shown in cyan sticks. The substrate mannobiose in (b) and (c) are shown as green stick, respectively. Other proposed substrate binding sites are showed light blue sticks (W104, R105, Y204, W282, D283 and Y300), magenta sticks (F110, K184 and N246), grey stick (D183), respectively.
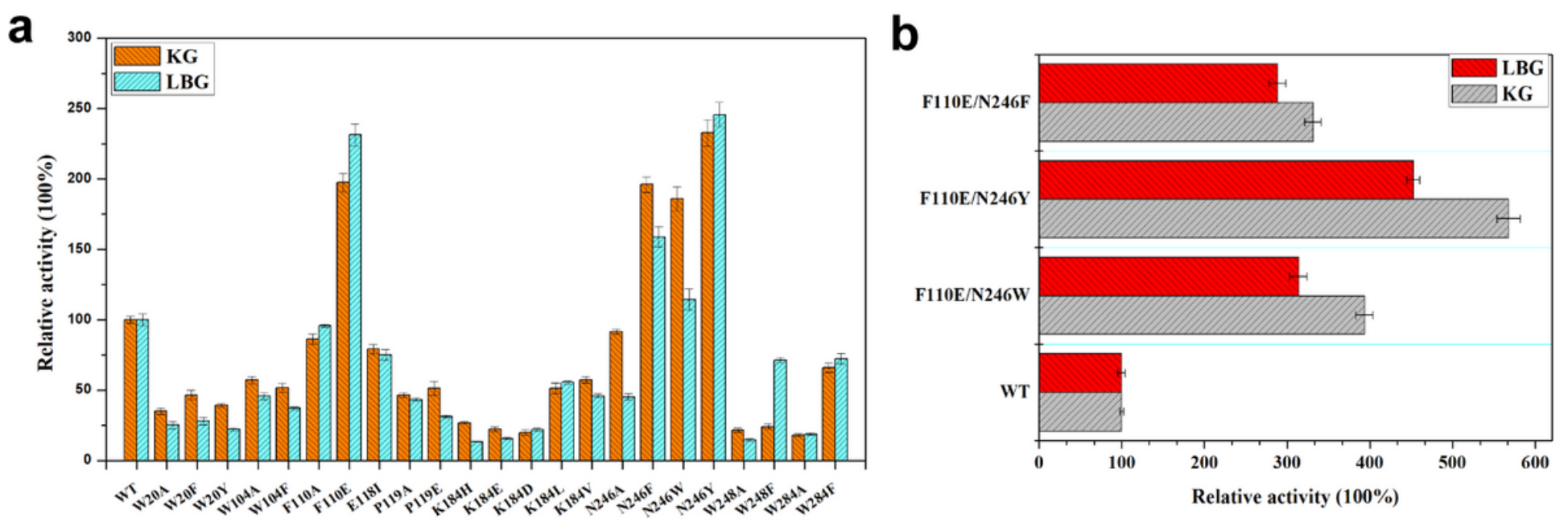

\section{Figure 5}

The catalytic relative activity of PcMan113-WT, mutants (a) and screened double mutants (b) towards KG and LBG. The catalytic activity of WT PcMan113 against KG and LBG is represented as $100 \%$ and the error bars are standard deviations $(n=3)$. 

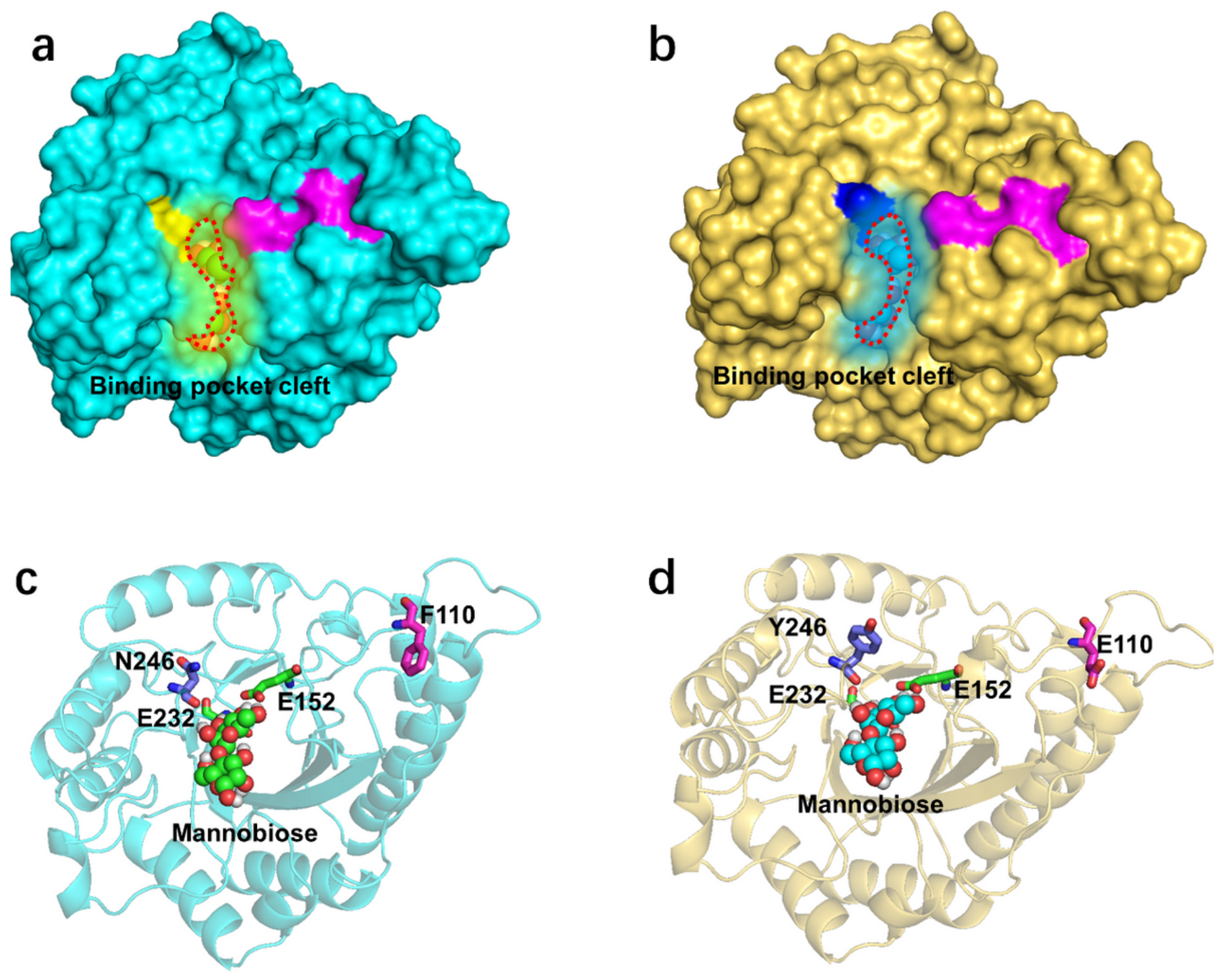

Figure 6

Binding modes and surface representation of the substrate in PcMan113-WT and PcMT3. The structures were obtained from cluster analysis of the MD trajectories during $30 \mathrm{~ns}$ of simulation. Surface representation of PcMan113-WT in color cyan (a) and PcMT3 in yellow (b) in complex with substrate mannobiose. The protein-substrate complex cartoon surface representation of PcMan113-WT in color cyan (c) and PcMT3 in yellow (d). The substrate binding channel surface was shown with red cycles in light yellow shadow (WT) and blue shadow (PcMT3). Site N246 is shown as a yellow surface and light blue sticks ((a) and (c)), site Y246 is shown as a blue surface and sticks ((b) and (d)), site F110 and E110 are shown as a magenta surface and sticks. The substrate is presented as green spheres. 


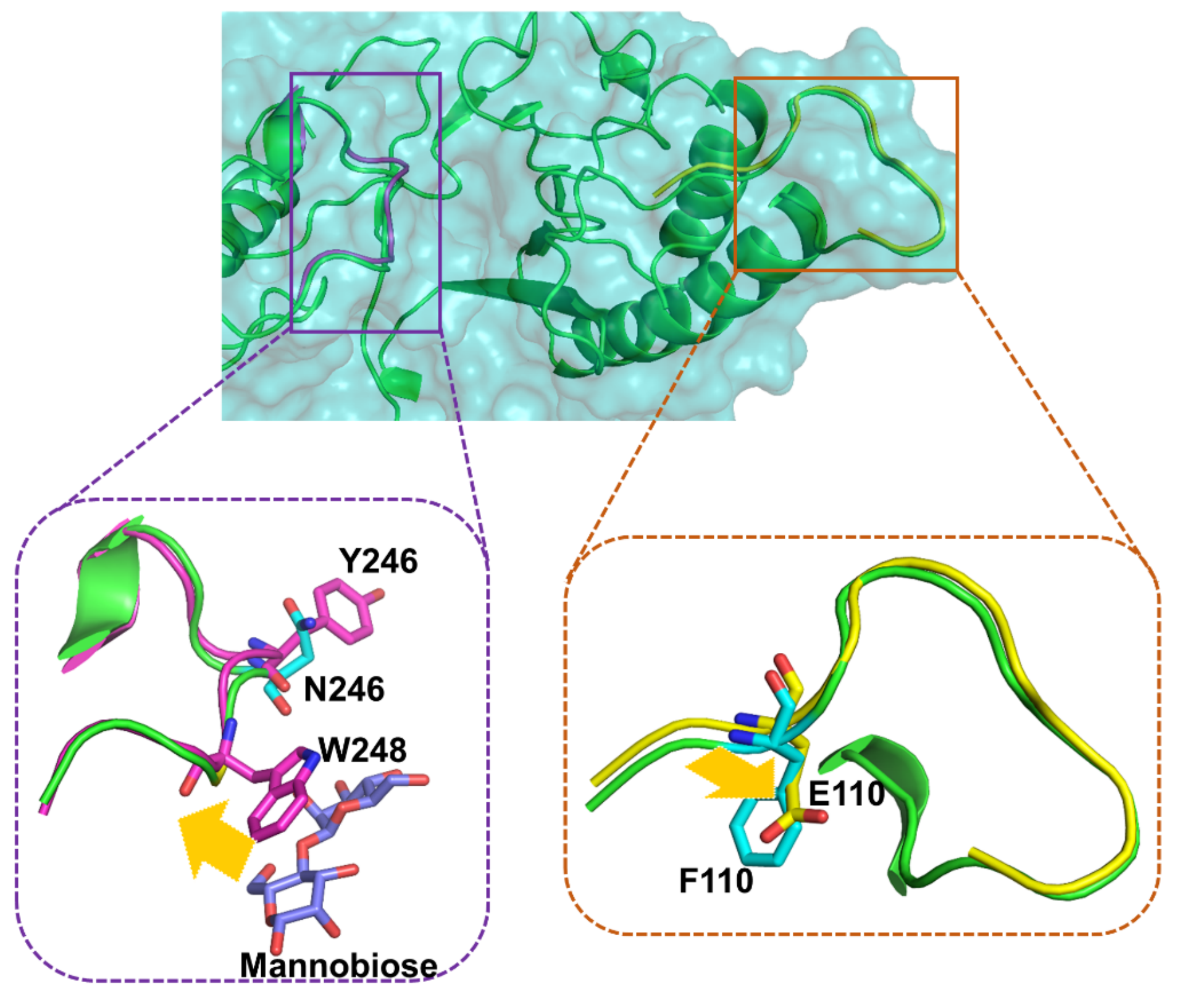

Figure 7

Ribbon representations of the MD-derived structures of PcMan113-WT and PcMT3 with substrate: (a) overall structural overlap of WT and PcMT3, (b) enlarged view of the loop R243-N255 (in color of magenta) in WT and MT3, and (c) enlarged view of the loop F110-E118(in yellow) in WT and PCMT3. PcMan113-WT is shown in green and cyan, respectively. Mannobiose is shown as light blue sticks.

\section{Supplementary Files}

This is a list of supplementary files associated with this preprint. Click to download.

- GRAPHICALABSTRACT.tif

- Supplementarylnformation.docx 